\title{
Study of System Intuition by Noetic Science Founded by QIAN Xuesen
}

\author{
Zhongtuo Wang \\ Institute of Systems Engineering, Dalian University of Technology \\ 116085 Dalian, China \\ wangzt@dlut.edu.cn
}

\begin{abstract}
This talk investigates the meaning, contents and characteristics of systems institution on the basis of Noetic Science, which was founded by Qian Xuesen. The systems intuition is the human capability to find the hidden system imagery of the object or to create an imagery of new system. The basic noetic foundation of system intuition and cultural influence to it are studied. The open problems are also listed.
\end{abstract}

Keywords: System intuition, Noetic Science, Imagery thinking, Inspiration, Tacit knowledge. 